\title{
A knowledge-based approach to wildlife management
}

\author{
Barbara Paterson ${ }^{1,2^{*}}$, Chris J. Brown ${ }^{3}$, Greg Stuart-Hill ${ }^{4}$, Heike Winschiers ${ }^{5}$, \\ Les G. Underhill', Tim T. Dunne ${ }^{6}$, Britta Schinzel ${ }^{7}$, Ben Beytell, \\ Fanuel Demas ${ }^{2}$, Pauline Lindeque ${ }^{2}$, Chris Weaver ${ }^{4}$ \\ Avian Demography Unit, Department of Statistical Science, University of Cape Town, Rondebosch, 7701 South Africa \\ ${ }^{2}$ Ministry of Environment and Tourism, Directorate of Scientific Services, Private Bag 13306, Windhoek, Namibia \\ ${ }^{3}$ Namibia Nature Foundation, P.O. Box 245, Windhoek, Namibia \\ ${ }^{4}$ World Wildlife Fund for Nature, Living in a Finite Environment (Life), P.O. Box 9681, Windhoek, Namibia \\ ${ }^{5}$ School of IT, Polytechnic of Namibia, Private Bag 13388, Windhoek, Namibia \\ ${ }^{6}$ Department of Statistical Sciences, University of Cape Town, Rondebosch, 7701 South Africa \\ 'Institut für Informatik und Gesellschaft, Albert-Ludwigs-Universität Freiburg,., Friedrichstr. 50, 79098 Freiburg im Breisgau, \\ Bundesrepublik Deutschland \\ ${ }^{8}$ Ministry of Environment and Tourism, Directorate of Parks and Wildlife, Private Bag 13306, Windhoek, Namibia \\ Received 16 July 2007. Accepted 18 March 2008
}

\begin{abstract}
We present a method for developing a knowledge base to give wildlife managers timely access to pertinent information. This knowledge-based approach to species management in Namibia focuses on anthologies and management strategies for high-value mammal species. To manage wildlife resources sustainably access to an appropriate knowledge base is essential. The strategic species management approach promotes planning on local, national, and international levels. A hypermedia Information System for Rare Species Management (IRAS) and a decision support system (Wildlife Introduction Advisor) are described. Unlike print media, which become dated, hypermedia is easily updated. The non-sequential nature of hypermedia allows information extraction according to the readers needs. Due to this flexibility hypermedia supports adaptive management and information sharing. Usability evaluation of the IRAS system disproved our assumption that established wildlife conservation categories such as habitat, distribution, and numbers are appropriate and sufficient to structure species information. This result led to a restructuring of the information according to management objectives. Evaluation of the Wildlife Introduction Advisor indicates that the model performs reliably and is robust towards input error. Human experts seem to be susceptible to bias. The economic benefits associated with wildlife translocations demand accountability through a well-defined, transparent distribution process. In Namibia this demand is achieved through the use of a knowledge-based decision support tool.
\end{abstract}

Key words: expert system, decision support, hypermedia system, knowledge-based system, natural resource management, wildlife management

\section{INTRODUCTION}

The multifaceted field of wildlife management embraces diverse issues such as habitat conservation, co-management, sustainable utilization, and political patronage. In order to adequately address complex decision scenarios, wildlife managers have to integrate knowledge from diverse disciplines, such as ecology, anthropology and economics. However, many of the wildlife departments in southern Africa have become staffed by young graduates with limited field-based expe-

\footnotetext{
*To whom correspondence should be addressed. Present address: Marine Research Institute and Zoology Department, University of Cape Town, Private Bag X3, Rondebosch, 7701 Cape Town, South Africa.E-mail: barbara@paterson.alt.na
}

rience. It has become apparent that there is a need to provide access to the existing stream of knowledge through both technology and the creation of a basis for communication and debate.

We present a method to develop a knowledge base for rare and high-value wildlife species that provides wildlife managers with timely access to pertinent information. This approach consolidates and systematizes the existing knowledge, while at the same time harnessing its diversity and variety. This knowledge-based approach to rare species management in Namibia focuses on compiling knowledge bases and developing appropriate computerized tools. The aim of this paper is to describe and evaluate this approach. 
Box 1. Specifications for a knowledge base for high-value species management in Namibia.

1. Biological Information:

a. Habitat

b. Food

c. Reproduction, including lifetime issues e.g. age and time period of reproduction; summary of important indicators in life cycle; specification of intrinsic rate of increase.

d. Cross species interaction, e.g. competition, predator-prey relations

e. Relevant behaviour, e.g. territorial behaviour, intra-specific aggression

f. Distribution of the Namibian meta-population, area carrying capacities, past, present and core ranges of all subpopulations and possibilities of future range expansion

g. Population abundance, relative estimates and population trends over time. Meta-description of all census data, with respect to survey method, sources of bias, statistical sampling errors and confidence intervals.

h. Taxonomic and genetic issues

i. Status of population based on IUCN categories

2. Conservation Significance: State whether the species is of national, regional, or global significance (or some combination thereof) and reasons, e.g. biodiversity and economics.

3. Factors limiting the species with respect to meta- and sub-populations within the area, e.g. habitat condition, availability and utilization; food specificity; human induced factors.

4. Threats to species, meta- and sub-populations and habitat.

5. Biological Management / Protection

a. Overview of conservation measures taken and proposed to protect the species with respect to meta- and sub-populations within the area

b. Identification of elements of best practice in the sub-region on which to base management options per species or species-groups

c. Identification of threats and opportunities for enhanced species conservation by implementing different transboundary collaborative initiatives

d. Description of captive breeding measures taken or proposed

e. Suitability of species for sustained harvesting

6. Stakeholding

a. Identification of stakeholders

b. Human activities that may negatively impact on the species

c. Opportunities and limitations for development

d. Support and incentive schemes in place and proposed to enhance stakeholder participation

\section{THE KNOWLEDGE-BASED APPROACH}

\section{Knowledge base development}

Compiling a knowledge base on rare and high-value species in Namibia requires a concept of information needs. Thus we developed explicit specifications describing the information required to improve the management of rare and high-value species in Namibia (Box 1). These specifications guided a series of studies, which collated information on 10 selected high value species, i.e. buffalo, Syncerus caffer caffer (Martin 2002); roan, Hippotragus equinus; sable, Hippotragus niger niger, and tsessebe, Damaliscus lunatus lunatus (Martin 2003); reedbuck, Redunca arundinum arundinum; waterbuck, Kobus ellipsiprymnus; lechwe, Kobus leche leche, and puku Kobus vardonii (Martin 2004); hippopotamus, Hippopotamus amphibius (Martin 2005a), and savanna elephant, Loxodonta africana (Martin 2005b). Each study provides a comprehensive overview for a species or species group, taking account of biological information, conservation significance, factors limiting the species, direct and indirect threats, management and protection of the species populations as well as identifying stakeholders and opportunities for transboundary collaboration with neighbouring countries. Each document provides an analysis of past and present management practices and offers recommendations for future management. This information was then used to develop management strategies for these 10 species. These strategies were documented in management plans (Ministry of Evironment and Tourism Namibia 2002, 2003, 2004, 2005a,b) based on a generic format (Box 2), which was adapted to the specific requirements of each species or species group. The drafting process of the species management plans involved wildlife management experts and scientists from government, 
Box 2. Generic management plan format for rare and high-value mammal species in Namibia.

EXECUTIVE SUMMARY

- Description of national species population within regional context

- Key issues and challenges

- Development process

- Time frame

- Diagram of logical framework

\section{INTRODUCTION \& BACKGROUND}

Conservation Status

- Present and desired conservation status of species and required intensity of management

\section{Populations}

- Description of meta-population of species

- Background and Rationale for Strategy

- Reasons why species is in need of management plan.

- The role of this management plan within annual planning cycle/5-year cycle

VISION

- A clear picture of the species long-term direction. (Requires knowledge why and to what purpose the species needs a management plan.)

\section{RISK AND ASSUMPTIONS}

- List risks and assumptions on which any conservation effort for this species is based.

TIMING AND DURATION OF STRATEGY

- e.g. 5 years

\section{STRUCTURAL FRAMEWORK FOR STRATEGIC OBJECTIVES}

Formulate objectives appropriate for the species under consideration

i. Strategic Objective

- Formulate statement to convert strategic vision into specific targets (results and outcomes)

- Must be possible to measure whether achieved or not

- Should be ambitious

ii. Rationale

- Background/context against which the objective has been developed

iii. Targets/Results

- List explicit targets, for specific time frame (short-term, mid-term, long-term)

iv. Strategies

- Steps necessary to achieve each target

v. Indicators for early warning

- Signal in case development is going in undesirable direction or stagnates

IMPLEMENTATION PROCESS \& UPDATE PROCEDURES

- Dates for review and circulation of draft strategy

- Revision and update procedures

- Guidelines for action planning

\section{ACKNOWLEDGEMENTS}

\section{REFERENCES}

GLOSSARY AND DEFINITION OF TERMS

ANNEXES

non-governmental organizations and universities. This diversity aimed at a high quality standard (Gerber \& Schultz 2001).

\section{An information system for rare species management}

Knowledge differs from data because it includes experience, interpretation and values. Such concepts cannot easily be divided into manageable units. The computerization of knowledge is there- fore a challenge. On the other hand almost anything can be expressed in natural language. Language can convey facts and data, values and perceptions, narratives and contexts. Natural language is thus a suitable medium of knowledge communication and the word processor is the de facto knowledge management tool for natural resource managers (Rauscher \& Reynolds 2003).

However, information that is contained in printed documents such as reports and journals is often 
not easily extractable. Print media are essentially linear, designed to be read sequentially with each idea based on previously covered ground. When consulting such documents, having to read the entire document is time-consuming. To accommodate the need for easy extraction, authors use meta-structures such as headings, table of contents, indexes and figures. Nonetheless, wildlife managers often require complex information that may not be found in a single document. Moreover, printed information is often dated because the updating of printed media is cumbersome. When part of a document has become dated reprinting has to wait until revision and update of the entire document is justified. When documents have been compiled as part of a project that has ceased to exist, it may not be clear where the responsibility to update the information lies. Electronic media, on the other hand, are easily updated and the example of the World Wide Web (WWW) demonstrates how a vast amount of information is cross-referenced into a single information source. Hypermedia, the format of the WWW, combines the advantages of natural language with the updating facility and dissemination power of electronic media.

Recognizing the limitations of a knowledge base in print format we developed a simple hypermedia information system (IRAS). This system eases information access and has the potential to develop into a knowledge management system (Paterson 2004). The species reports and management plans were analysed in terms of recurring themes to identify knowledge categories. The content was then edited into concise information units, which were converted into hypermedia documents. Each unit is self-contained and independently understandable, similar to tables and figures in a journal article. As web pages, all units are cross-referenced by hyperlinks to form a coherent and structured network.

\section{Wildlife Introduction Advisor}

One common result of the 10 species studies is the recommendation to translocate animals from areas of abundance to other areas within their historical range. In the past, wildlife in Namibia was reduced to the point that many areas today have little wildlife left. But since Namibian independence in 1990, the demand for tourism, game farming and sport hunting has increased. Thus land is becoming available for the reintroduction of wildlife. The growth in communal conservancies presents Namibia with a twofold conservation opportunity: by re-establishing wildlife species into their former ranges opportunities are being created for local communities to improve their livelihoods through sustainable utilization of animals for tourism, trophy hunting and meat.

Choosing appropriate conservancies for reintroduction of particular species, however, involves complex multi-criteria decision analysis (MCDA) and many of the issues are politically charged. To provide transparency and to guide the decisionmaking process a decision support system 'Wildlife Introduction Advisor' (in the following referred to as IntroAdvisor) was developed using NetWeaver fuzzy logic modelling software (Miller \& Saunders 2002). For a summary of key concepts and constructs related to the design and use of NetWeaver knowledge bases see Reynolds et al. (2000). This decision support system was developed in consultation with wildlife management experts. The goals and criteria for translocating wildlife into communal conservancies were structured hierarchically. Broad top-level goals were systematically broken down into increasingly precise sub-goals and finally resulted in a set of translocation criteria. For a description of how this structure was developed see Paterson et al. (in press). The criteria form the input variables for the decision support system and include historic range, access to water, and management capacity of the conservancy, existing land uses, and threats to the species, tourism potential, utilization potential, and impact on livelihoods. IntroAdvisor evaluates the suitability of each conservancy for 22 species based on the objectives hierarchy and input values elicited from experts. The results are visualized as bar charts (Fig. 1) and maps which allow for comparison on a national scale (Fig. 2). These outputs indicate which species can be translocated into a specific area and which areas are suitable for a given species. In addition it is shown how each area is evaluated in terms of each criterion.

\section{Evaluation of the tools}

\section{Usability test of the hypermedia information system IRAS}

After completion of a prototype, the IRAS hypermedia system was evaluated by wildlife managers and scientists from the Namibian Ministry of Environment and Tourism and software usability experts from the School of Information Technology of the Polytechnic of Namibia. For a 


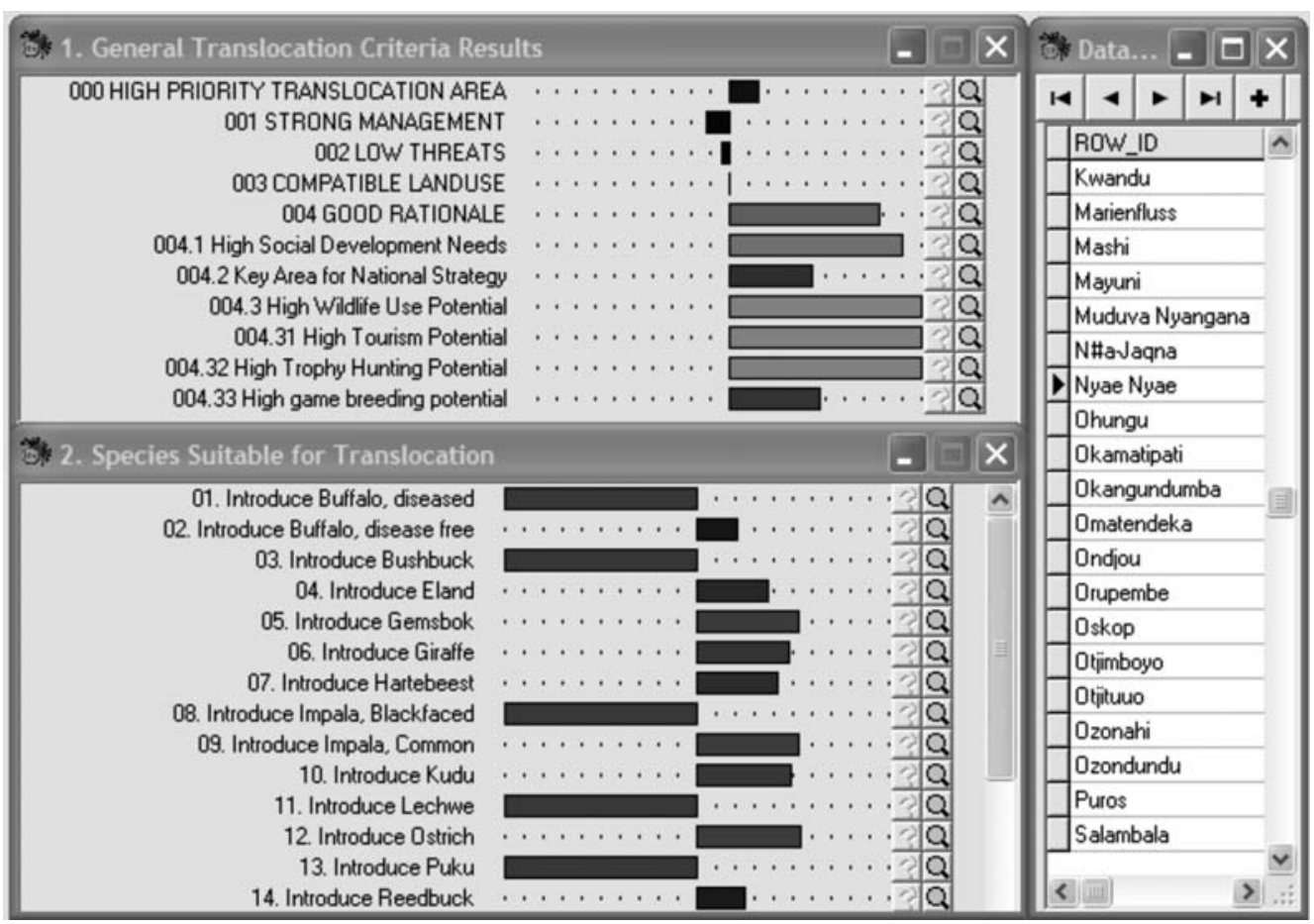

Fig. 1. Wildlife Translocation Advisor results visualized on an individual conservancy scale. The top chart indicates to which extent the selected conservancy fulfils individual translocation criteria. The lower chart indicates to which extent each species under consideration is suitable for translocation into the selected conservancy. Negative values are represented in shades of red and extend to the left, positive values are represented in shades of green and extend to the right.

description of the usability evaluation in a cross-cultural setting see Winschiers \& Paterson (2004). Two main usability issues were identified: 'ease of orientation' and 'unexpected results' (Table 1). The evaluation revealed structural and conceptual weaknesses in the way the information was organized both in the printed documents as well as in the prototype information system. The assumption that categories, such as habitat, distribution, and numbers are well established in the

Table 1. IRAS evaluation results. Testers performed task-solving exercises. Usability data were obtained through observations and questionnaire $(n=11)$.

\begin{tabular}{lcccc}
\hline Data obtained from questionnaires & No data & Low & Medium & High \\
\hline Ease of of use & 2 & 1 & 5 & 7 \\
Ease of orientation & 2 & 0 & 4 & 1 \\
Expectations of results of operations are met & 3 & 0 & 2 & 6 \\
Ease of learning to use the system & 4 & 0 & 5 & 7 \\
Accessability & 2 & 2 & 2 & 7 \\
Adequacy of information & 2 & 0 & 2 & 2 \\
Adequacy of subject related terminology & 2 & & 5 \\
\hline
\end{tabular}

Data obtained from observations

\section{Ease of orientation}

Expectations of results of operations are met

Ease of task completion

User's ease of learning

$\begin{array}{ll}0 & 2 \\ 4 & 1 \\ 0 & 4 \\ 3 & 2\end{array}$

5

0 


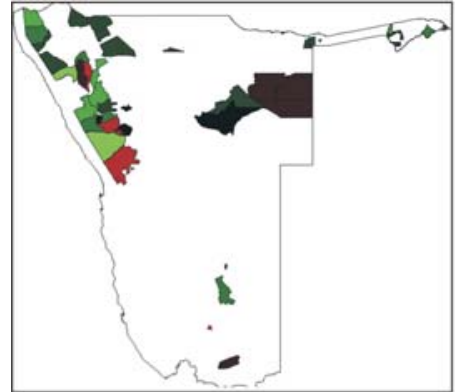

(a)

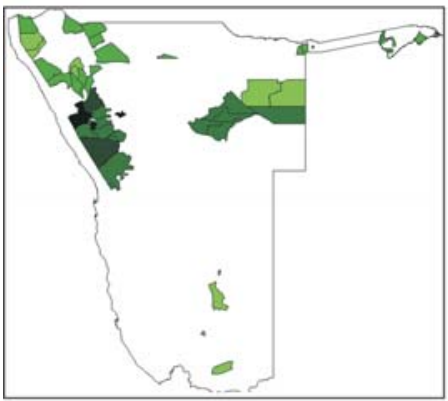

(d)

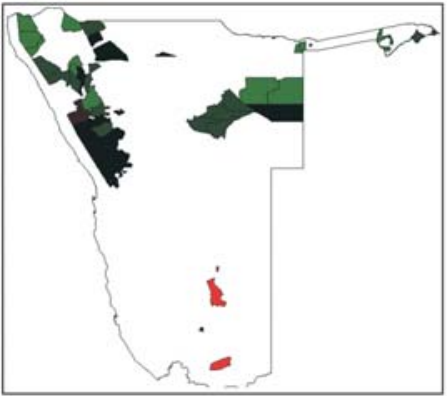

(g)

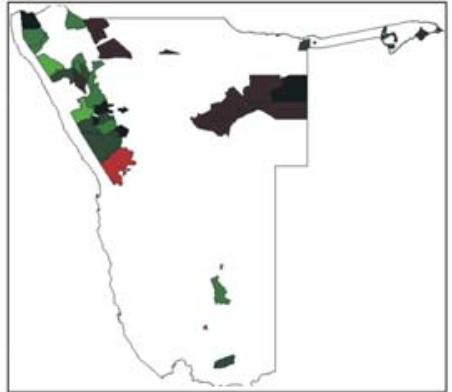

(b)

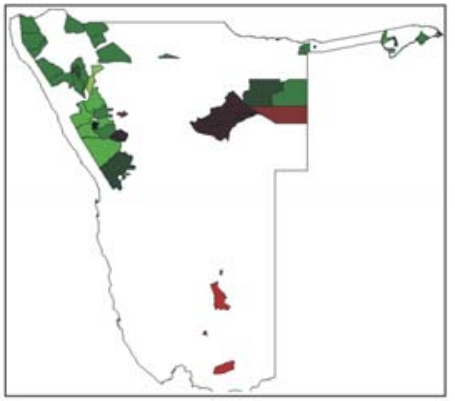

(e)

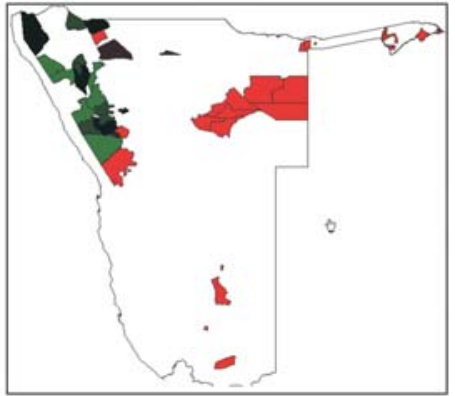

(h)

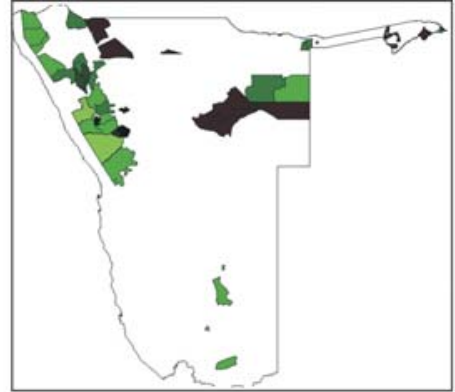

(c)

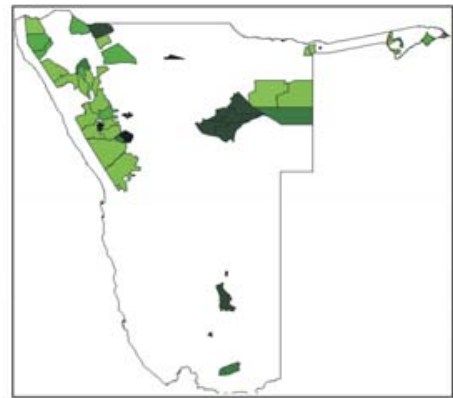

(f)

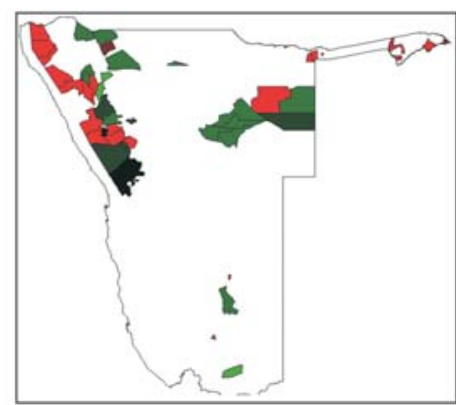

(i)

Fig. 2. Wildlife Translocation Advisor results visualized on a national scale. The maps indicate the fulfilment of individual criteria, e.g (a) high management capacity, (b) low threats, (c) compatible land use, (d) high social development needs, (e) contribution to national conservation strategies, (f) high utilization potential or the suitability of each are for a particular species, e.g. (g) giraffe, Giraffa camelopardalis; (h) black-faced impala Aepyceros melampus petersi, (i) springbok, Antidorcas marsupialis. Truth values are displayed in degrees of green (true) and red (false).

wildlife conservation community and must therefore be appropriate and sufficient to structure the species information proved to be wrong. Although familiar with the terminology, users experienced difficulty in finding specific information because it was categorized in unexpected ways. As a result of the usability evaluation the IRAS content was re-analysed and a knowledge organization system for the information was developed. The information units are of different formats, such as text, maps, tables and figures. The new scheme arranges this heterogeneous information accord- ing to species, theme, and sub-theme (Fig. 3). The navigation system, i.e. the labels and cross references, links the information categories to management objectives. The start page for each species organizes the objectives according to thematic groups, such as habitat objectives, economic objectives and social objectives. From here the user has access to a description of management actions that may help to achieve the objective or to background information which forms the rationale for the particular objective (www.nnf.org.na/ RARESPECIES/infosys/index.html). 


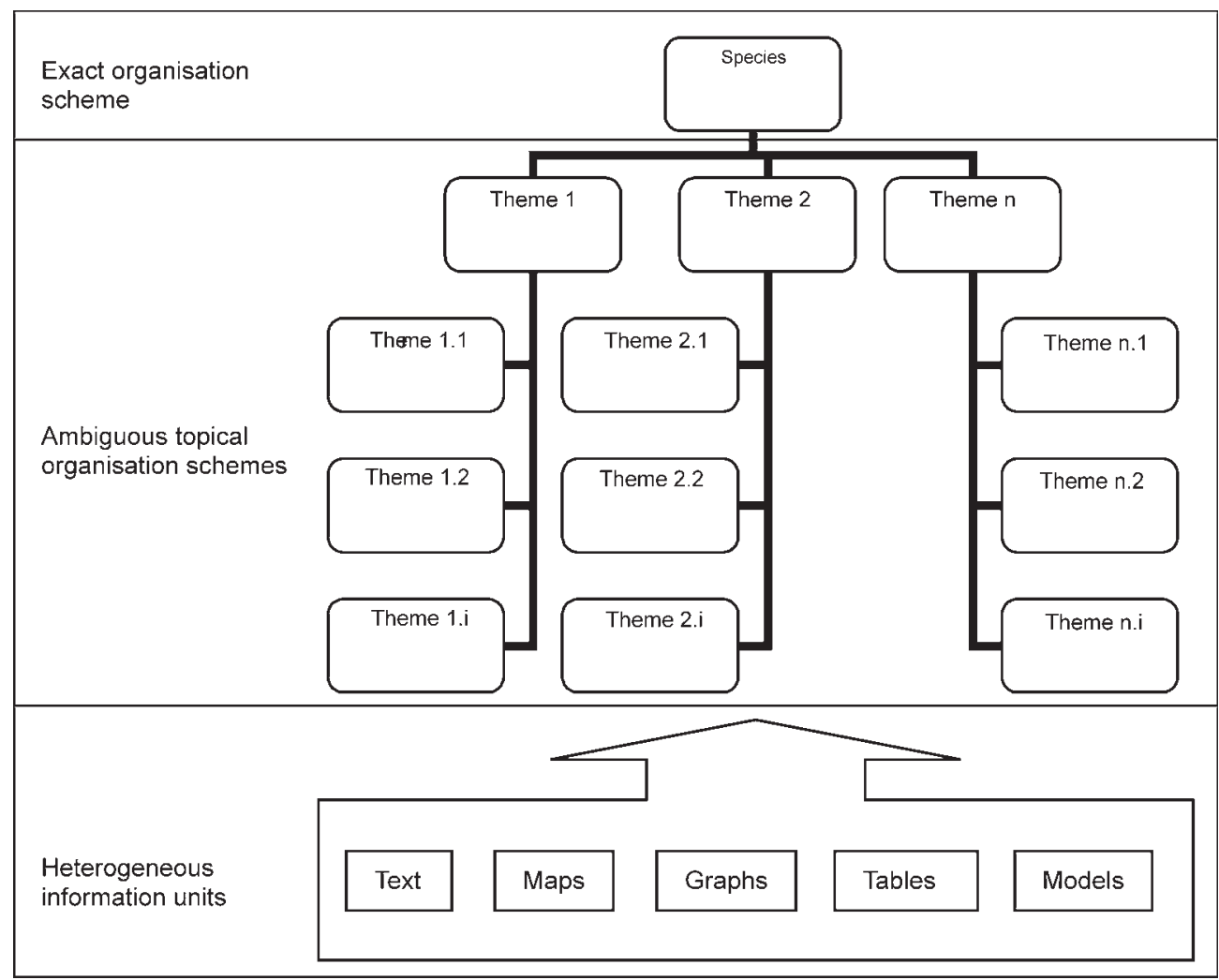

Fig. 3. IRAS organization system for wildlife management knowledge. Information is formatted in different ways and organized by species. For each species different themes and sub-themes categorize the information further.

\section{Evaluation of the decision support system (IntroAdvisor)}

To evaluate the validity of results produced by the IntroAdvisor model, a group of experts scored the overall suitability of each conservancy for a number of species. The resulting 600 scores were compared with the results computed by the model. In $82 \%$ of the cases the expert assessment agreed with the model results. This evaluation indicates that the model performs reliably and satisfactorily. A more detailed analysis (Paterson et al., in press) suggests that experts are likely to focus on some criteria while ignoring others. This suspicion was later confirmed by one of the experts who had participated in the evaluation (P. du Preez, pers. comm.). These examples may be indicative of the limitations of unstructured ad hoc decision-making (Linkov et al. 2006). Sensitivity analysis showed that although model variables are based on expert opinion model outputs are robust towards input error (Table 2, Paterson et al., in press). After a three-year review process during which the

Table 2. Sensitivity of IntroAdvisor results to changes in input. The values are average changes in output after changing input variables by one scale unit. Results for three tests are shown: (i) each variable was changed one at a time, while all other variables remained constant; (ii) two variables at a time were changed, while all other variables remained constant; (iii) All variables were incremented simultaneously by one scale unit. One unit on the input scale corresponds with 0.200 on the output scale.

\begin{tabular}{lcccc}
\hline & $\begin{array}{c}\text { Disease-free buffalo } \\
\text { (Syncerus caffer) }\end{array}$ & $\begin{array}{c}\text { Red hartebeest } \\
\text { (Alcelaphus buselaphus) }\end{array}$ & $\begin{array}{c}\text { Gemsbok } \\
\text { (Oryx gazella) }\end{array}$ & $\begin{array}{c}\text { Roan } \\
\text { (Hippotragus equines) }\end{array}$ \\
\hline Individual variables & 0.005 & 0.012 & 0.010 & 0.014 \\
Variable pairs & 0.013 & 0.014 & 0.019 & 0.024 \\
All variables & 0.092 & 0.205 & 0.201 & 0.238 \\
\hline
\end{tabular}


IntroAdvisor input data have been updated annually and outputs have been circulated for review, the system is now being used to guide game introduction decisions in Namibia.

\section{DISCUSSION}

The method presented here towards enhancing rare and high value species management is a knowledge-based formula. At the centre lies the development of appropriate knowledge repositories as well as the procurement of knowledgebased decision support within a specific decisionmaking scenario. Our approach is inclusive and exploratory, building from one process-based output to feed into the next.

\section{Conservation management planning}

Rare and high-value species are an important resource. To manage this resource sustainably information and knowledge are essential. An anthology of all available information on the past, present and core ranges of the selected species, their status in different areas as well as trend data, habitat requirements and management options is thus beneficial.

The national species strategies are not to be confused with a single species conservation approach. The species management plans define management strategies that overlap from one species to another. Developing management strategies requires the definition of objectives and targets for each species regarding both biodiversity and economic goals. The management plans thus provide explicit guidance and targets for local planning. At the time of this study, conservancies in Namibia were in the process of developing local management plans, and protected areas (parks) were doing the same. As part of these processes, management practices that were identified within the species management plans were incorporated into local management plans. These strategies were operationalized through annual work plans and are part of ongoing local management practice including monitoring, reporting and adaptive management responses. Thus the strategic species management approach promoted conservation management planning on both national and local levels.

\section{Transboundary collaboration}

At the regional, transboundary level, the species studies and the drafting of national species management plans for Namibia provided an opportunity to initiate collaboration between Namibia and its neighbours, Botswana in particular. Two workshops took place bringing together key stakeholders from Namibia and neighbouring Botswana. During these workshops the knowledge specifications put forward by Namibia were reviewed and priority species, i.e. their needs and management requirements, and opportunities for transboundary collaboration were identified and discussed and those species studies and draft management plans that had been completed were reviewed. In this way information needs were made explicit and available expertise was pooled together to build capacity. Thus by developing a national vision while at the same time sharing information and maintaining dialogue each country contributed towards the emergence of a larger, region wide vision that exceeded national boundaries.

\section{Hypermedia supports adaptive management}

The species anthologies and draft management plans contain pertinent descriptive and strategic knowledge regarding each species. However, paper-based documents are subject to several constraints. The species documents are no exception. Later sections of the documents build on information presented in earlier sections. Although some information is summarized in tables and figures for easy extraction, other information is 'buried' in the text. To extract the complete host of information, each document has to be read from beginning to end, a procedure which may be too time-consuming for decision-makers (Pullin et al. 2004). The IRAS knowledge repository on the other hand is a highly structured collection of autonomous information units, which can easily be extracted in any order that is most appropriate to the user's needs.

Much of the content of the background studies and management plans relies on dated information. The flexibility of the medium allows the ongoing inclusion of new insights and information which are generated by monitoring the success and failure of management measures. A simple hypermedia system such as IRAS is thus a valuable tool for supporting and documenting adaptive management processes (Paterson 2004).

\section{Decision support systems to enhance problem-solving power}

The objective of IRAS is to provide easy access to a wide range of information on Namibia's rare 
and high value wildlife species. However, the generality of this medium, which allows representing almost any knowledge, sacrifices precision. Although IRAS provides a host of background information that is valuable to decision-makers, the system itself has only low problem-solving power (Rauscher \& Reynolds 2003). The Wildlife Introduction Advisor on the other hand, has a narrower, specific knowledge focus. The system supports decisions that are linked to two specific questions: Which conservancies are suitable for the introduction of a given species? And which species can be introduced into a given conservancy? Although this knowledge scope is much narrower, finding answers to these questions is a complex decision problem, which requires the careful weighing of multiple criteria. The NetWeaver software environment is useful for developing large knowledge bases for complex decision problems (Miller \& Saunders 2002). The validity tests and sensitivity analyses show that this approach is appropriate to aid the selection of translocation sites for wildlife species (Paterson et al., in press).

\section{CONCLUSION}

The economic benefits associated with wildlife translocations demand that these benefits are shared in a well-defined, transparent manner that enhances accountability (Roe et al. 2000). The main value of a knowledge-based decision support tool such as Wildlife Introduction Advisor lies in its standardization of the decision-making process. Such a tool prescribes an agreed upon set of criteria and ensures that all criteria are equally considered.

Due to its flexibility, a simple hypermedia system such as IRAS can be used to improve knowledge management within wildlife institutions as well as to improve information sharing between institutions. The decision support system 'Wildlife Introduction Advisor' demonstrates a transparent and rigorous approach to providing support for multicriteria decision problems in natural resource management.

In Namibia, with the aid of these systems, the relevant knowledge about species management can more easily be communicated both to the local level to influence management of protected areas or communal lands as well as to the national and regional levels to contribute to a strategy that transcends international boundaries between neighbouring countries.

\section{ACKNOWLEDGEMENTS}

This publication was made possible through support provided by the Namibia Nature Foundation, the Ministry of Environment and Tourism, and Worldwide Fund for Nature (WWF, Namibia) Living in a Finite Environment (LIFE) Programme through funding from the United States Agency for International Development (USAID) Namibia mission under the terms of the Cooperative Agreement No. 690-A-00-99-00227-00. The views expressed in this document are the views of the authors and are not necessarily those of USAID.

\section{REFERENCES}

GERBER, L.R. \& SCHULTZ, C.B. 2001. Authorship and the use of biological information in endangered species recovery plans. Conserv. Biol. 15: 1308-1314.

LINKOV, I., SATTERSTROM, F.K., KIKER, G., BATCHELOR, C., BRIDGES, T. \& FERGUSON, E. 2006. From comparative risk assessment to multi-criteria decision analysis and adaptive management: Recent developments and applications. Environt. Int. 32: 1072-1093.

MARTIN, R.B. 2002. Species report for southern savanna buffalo Syncerus caffer. Ministry of Environment and Tourism, Windhoek, Namibia. Online at http://www. rarespecies.org.na/library/species reports.htm.

MARTIN, R.B. 2003. Species report for roan Hippotragus equinus, sable Hippotragus niger and tsessebe Damaliscus lunatus. Ministry of Environment and Tourism, Windhoek, Namibia. Online at: http://www. rarespecies.org.na/library/speciesreports.htm.

MARTIN, R.B. 2004. Species report for southern reedbuck Redunca arundinum, common waterbuck Kobus ellipsiprymnus, red lechwe Kobus leche and puku Kobus vardoni. Ministry of Environment and Tourism, Windhoek, Namibia. Online at: http://www. rarespecies.org.na/library/speciesreports.htm.

MARTIN, R.B. 2005a. Species report for hippopotamus Hippopotamus amphibius. Ministry of Environment and Tourism, Windhoek, Namibia. Online at: http:// www.rarespecies.org.na/library/speciesreports.htm.

MARTIN, R.B. 2005b. Species report for savanna elephant Loxodonta africana. Ministry of Environment and Tourism, Windhoek, Namibia. Online at http:// www.rarespecies.org.na/library/speciesreports.htm.

MINISTRY OF EVIRONMENT AND TOURISM NAMIBIA. 2002. Draft strategic species management plan for southern savanna buffalo Syncerus caffer. Ministry of Environment and Tourism, Windhoek, Namibia.

MINISTRY OF EVIRONMENT AND TOURISM NAMIBIA. 2003. Draft strategic species management plan for roan Hippotragus equinus, sable Hippotragus niger and tsessebe Damaliscus lunatus. Ministry of Environment and Tourism, Windhoek, Namibia.

MINISTRY OF EVIRONMENT AND TOURISM NAMIBIA. 2004. Draft strategic species management plan for southern reedbuck Redunca arundinum, common waterbuck Kobus ellipsiprymnus, red lechwe Kobus leche and puku Kobus vardoni. Ministry of Environ- 
ment and Tourism, Windhoek, Namibia.

MINISTRY OF EVIRONMENT AND TOURISM NAMIBIA. 2005a. Draft strategic species management plan for hippopotamus Hippopotamus amphibius. Ministry of Environment and Tourism, Windhoek, Namibia.

MINISTRY OF EVIRONMENT AND TOURISM NAMIBIA. 2005b. Draft strategic species management plan for savanna elephant Loxadonta africana. Ministry of Environment and Tourism, Windhoek, Namibia.

MILLER, B.J. \& SAUNDERS, M.C. 2002. NetWeaver Reference Manual: a compendium of NetWeaver and NetWeaver related terms, concepts and functions. Penn State University. Online at: http//www.rules-ofthumb.com.

PATERSON, B. 2004. Hypermedia as a knowledge management tool in wildlife management. Game Wildlife Sci. 21: 757-765.

PATERSON, B. In press. A fuzzy decision support tool for wildlife translocations into communal conservancies in Namibia. Environ. Modell. Softw.

PULLIN, A.S., KNIGHT, T.M., STONE, D.A. \& CHARMAN, K. 2004. Do conservation managers use scientific evidence to support their decision-making?
Biol. Conserv. 119: 245-252.

RAUSCHER, H.M. \& REYNOLDS, K.M. 2003. Natural resource management. In: Encyclopedia of information systems, Volume 3 (pp. 239-265). Elsevier Science, Bakersfield.

REYNOLDS, K.M., JENSEN, M., ANDERSEN, J. \& GOODMAN, I. 2000. Knowledge-based assessment of watershed condition. Comput. Electron. Agr. 17 315-333.

ROE, D., MAYERS, J., GRIEG-GRAN, M., KOTHARI, A., FABRICIUS, C. \& HUGHES, R. 2000. Evaluating eden: Exploring the myths and realities of community-based wildlife management: series overview. Evaluating Eden series no 8. A publication of the Biodiversity and Livelihoods Group (BLG). Online at: http://www.iied.org.

WINSCHIERS, H. \& PATERSON, B. 2004. Sustainable software development. In: G. Marsden, P. Kotzé \& A. Adesina-Ojo (Eds.), Fulfilling the promise of ICT, Proceedings of the 2004 annual research conference of the South African Institute of Computer Scientists and Information Technologists on IT research in developing countries (pp. 111-115). SAICSIT, Stellenbosch, South Africa.

Corresponding Editor: G.I.H. Kerley 